\title{
AGNJET v2.0: Towards self-consistent MHD+RT jet models for XRBs and AGN
}

\author{
Chiara Ceccobello* \\ Anton Pannekoek Institute for Astronomy, University of Amsterdam Netherlands \\ E-mail: c.ceccobello@uva.nl
}

\section{Sera Markoff}

Anton Pannekoek Institute for Astronomy, University of Amsterdam Netherlands

E-mail: s.b.markoffeuva.nl

\section{Riley Connors}

Anton Pannekoek Institute for Astronomy, University of Amsterdam Netherlands

E-mail: r.m.t.connors@uva.nl

Collimated outflows are a common feature observed in many astrophysical systems including Xray binaries (XRBs), active galactic nuclei (AGN), young stellar objects (YSOs) and gamma ray bursts (GRBs). Emission from such jets extends over the whole electromagnetic spectrum and is a proxy of the nature and activity of the central engine. To constrain the physics of jets both magneto-hydrodynamical (MHD) and radiative transfer (RT) calculations are needed. However, numerical simulations cannot handle full MHD+RT calculations on a useful timescale for fitting procedures. Hence, to fit and interpret observational data, semi-analytical models are required, such as the improved AGNJET code that we will discuss here.The AGNJET code [1] evaluates the emission from a multi-zone jet consisting of a nozzle followed by a quasi-isothermal conical expanding region. During the expansion a portion of the electrons can be re-accelerated, through a process that commences at a distance from the black hole (BH) determined by observational constraints. In this updated version of AGNJET ( [2], [3], hereafter CC17b and CR17, respectively), the inverse Compton component is evaluated allowing for multiple inverse Compton scatterings and pair production/annihilation. Together with other upgrades like electron cooling, such new features can help break the degeneracy between synchrotron-dominated and Compton-dominated spectral energy distributions of black hole disk/jet systems. I will also discuss the coupling of AGNJET with the semi-analytic, relativistic MHD model developed by [4-6] and [7, hereafter CC17a], aimed at constraining a subset of the input parameters based on self-consistent MHD calculations for the jet nozzle.This new version of AGNJET calculates the jet velocity profile and geometry self-consistently with ideal-MHD and includes a more accurate treatment of jet morphologies and dynamics, and the relevant physical processes.

11th INTEGRAL Conference Gamma-Ray Astrophysics in Multi-Wavelength Perspective 10-14 October 2016

Amsterdam, The Netherlands

\footnotetext{
* Speaker.
} 


\section{Introduction}

Black holes (BHs) are subjects of a great deal of fruitful astrophysical phenomena, heavily shaping the systems they belong to through accretion/ejection of the available matter. Due to their large mass span, from stellar mass to billions of solar masses, BHs are found in systems of very different scales, such as X-ray binaries (XRBs) and active galactic nuclei (AGN). Material provided by a donor star or by the innermost regions of galaxies feeds the nearby $\mathrm{BH}$ through an accretion disk structure. A significant fraction of the accretion power can be carried away by collimated, relativistic outflows, and deposited into the surroundings, affecting the environment up to very large distances from the BH (e.g. [8] and [9] for examples of effects at different scales). The accretion disk and associated jets emit radiation over the entire electromagnetic spectrum, from radio to $\gamma$-rays, exchanging the dominant role across different states of their evolution (e.g. [10]). XRBs are subject to rapid changes over timescales of days to months, while AGN are thought to go through equivalent stages over cycles of up to potentially millions of years [11]. Dedicated multiwavelength observing campaigns have shown us that XRBs and AGN exhibit many of the same features, as suggested and tested by [12], [13] and [14], among others, indicating that the physics of accretion may be mass-invariant. This is driving an effort focused on developing models capable of describing both XRBs and AGN. Accretion/ejection phenomena can be modelled with general relativistic magneto-hydrodynamic (GRMHD) simulations (e.g. [15]), although the lack of crucial ingredients such as a basic radiative treatment makes a direct test against observational data a very hard goal to achieve. On the other hand, several semi-analytical models for emission from disk/jet have been proposed, such as [16], [17], [18], [19], however none of them contain a self-consistent treatment of the geometry and the dynamics through MHD calculations. Therefore, a natural alternative comes from semi-analytical relativistic MHD (RMHD) models ( [4-6], SSCC17a) coupled with fairly accurate radiative transfer models, which can handle spectral fitting within a reasonable computational time.

We describe here the most recent developments of the AGNJET model for jet emission. First presented in [20], the model was originally based on a series of papers [21-23] providing the dynamics, and later upgraded in [1] and [24]. AGNJET has been successfully applied in multiwavelength spectral fitting of several XRBs and LLAGN, as in the recent [25,26] and CR17. Further changes to basic assumptions of the model have been described in Crumley et al. 2017, from here on C17, as well to the radiative processes happening in the jet in CR17 and CC17b.

\section{Model development}

In the original version of the AGNJET model, mildly-relativistic, bipolar jets are launched from the immediate proximity of the $\mathrm{BH}$, As the thin outer disk transition into a thick inner disk, the outflows are generated at some radius close to the inner part of the thicker disk, as seen in simulations. The jets receive a fraction of the total accretion power, assumed to be roughly equally divided between internal (magnetic and thermal) and kinetic (cold protons) contributions. The details of the disk/jet connection are largely unknown, therefore the base of the jet is defined as a collimated, cylindrical nozzle region acting as a corona, where ionized relativistic plasma resides. From the sonic nozzle free electrons are driven away from the compact object whilst they cool via syn- 

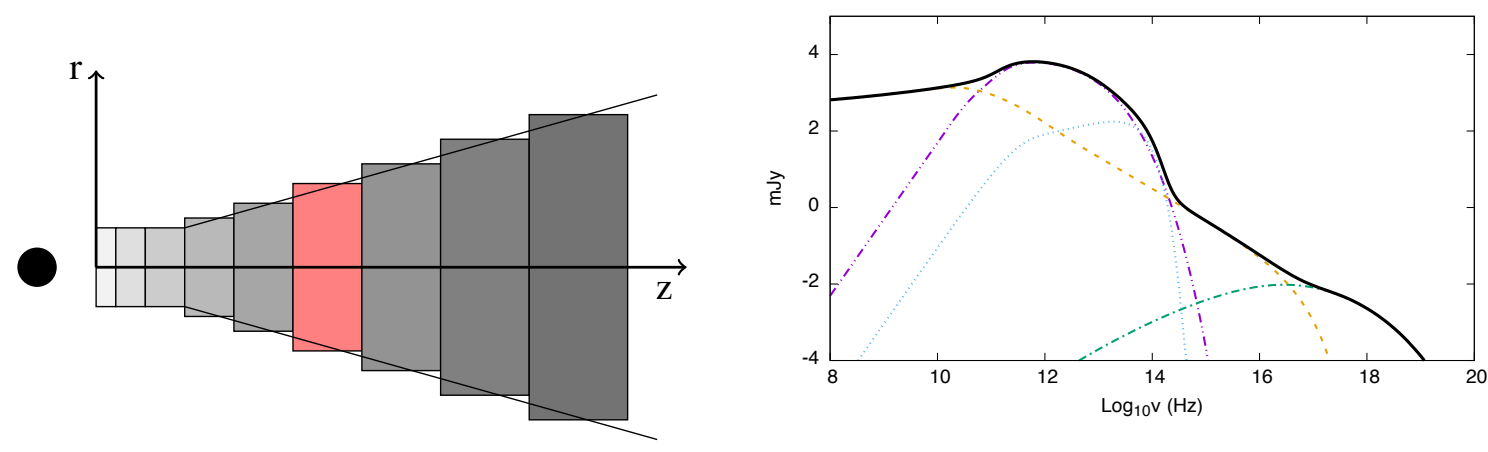

Figure 1: Left: Schematic view of AGNJET 's morphology. At the base there is a cylindrical nozzle, then the jet starts to expand almost freely. The region upstream with respect to the shock (red segment) is referred to as pre-shock region, while the segments located downstream are collectively called post-shock region. The extent of the nozzle as well as the position of the shock are free parameters of the model. Right: Components of AGNJET . The total spectrum (solid black line) is composed by several components: a preshock synchrotron spectrum (dot-dot-dashed purple line), a synchrotron-self Compton (dot-dashed green line), a post-shock synchrotron (dashed yellow line) and a thermal disk component (dotted blue line).

chrotron radiation and synchrotron-self Compton (SSC). Beyond the nozzle, the jet is expanding almost freely whilst maintaining a quasi-constant temperature. Although the mechanism responsible for keeping the jet in a quasi-isothermal state for its entire extent has yet to be determined (e.g. [27]), it is treated as an assumption motivated by observational requirements, as described in C17.

In order to explain the appearance of an optically thin power-law of particles, a shock region is invoked, the location of which is a free parameter in the model, determined by fitting to the data. Within the shock region a fraction of the electrons are reaccelerated into a power-law while radiating non-thermal synchrotron photons. The jet is divided up into segments (Fig. 1, left panel) and the final spectrum is calculated as a sum of the contribution of each segment. The total spectrum is formed by the several radiative processes at play (Fig. 1, right panel). Although already relatively complex, AGNJET was lacking several key features required to make it more physically accurate, such as the effect of electron cooling on the post-shock synchrotron radiation which has been implemented only recently by CR17. Another key limitation of the current model exists in the single-scattering limit of the SSC process and the absence of an even rough estimation of the contribution of pair creation/annihilation near the base of the jet. It is worth noticing that a partial treatment of the pairs was introduced by [24], but then dropped in later versions of the model because it was found to be relevant only for high-luminosity sources.

In the forthcoming paper by $\mathrm{CC} 17 \mathrm{~b}$, we remove the single-scattering limitation and we reinstate the estimation of pair production and annihilation rates as it was in [24]. We define the photon spectrum emerging from a jet segment, $i$, filled by hot leptonic plasma, as the sum of $k$ single-scattering Comptonization orders as follows

$$
f(x)_{i}=\sum_{k}^{\infty} f_{i, k}(x)
$$

where each term $f_{i, k}(x)$ is the spectrum of the $k$-scattered photons produced in the $i$ th-segment [28]. 

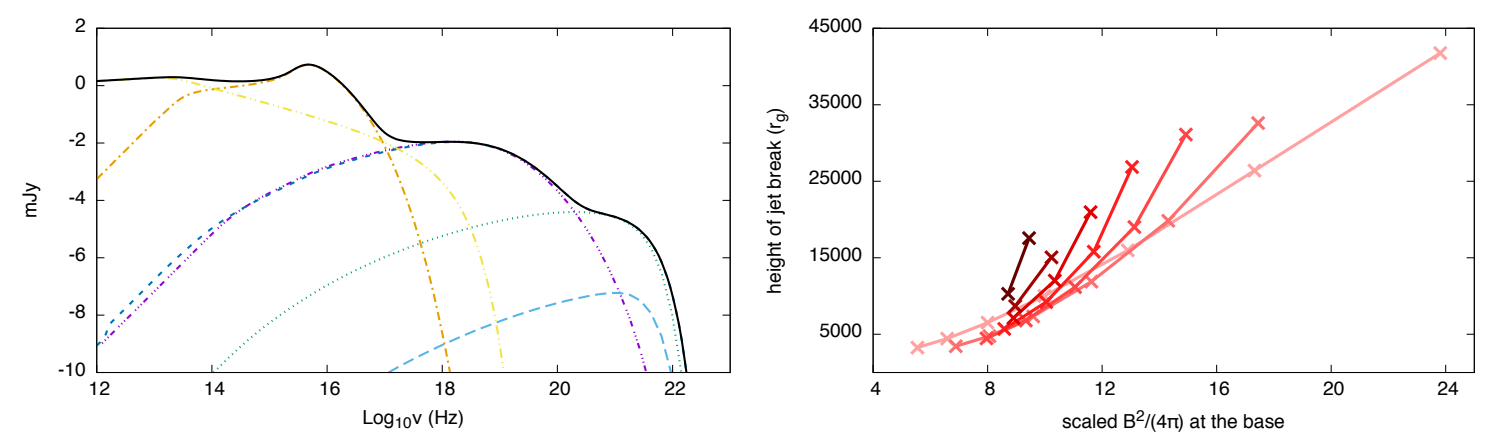

Figure 2: Left: An example of the different output spectra for the two operation modes, multiple (dot-dotdot-dashed purple line) and single scattering (dashed blue line), for the same set of input parameters. The dotted green line and the long-dashed light blue line are the second and third orders, respectively. The dashed dark blue line is the total SSC spectrum, while the dot-dashed orange line is the pre-shock synchrotron, the dot-dot-dashed yellow line is the post-shock synchrotron and the solid black line is the total spectrum. Right: Height of the jet spectral break as a function of the magnetic energy density at the base of the jet. Possible jet solutions are shown as crosses. For clarity, we only show a small subset of solutions (further solutions exist on finer scales, as well as for different combinations of jet parameters). Separate lines represents different efficiencies for channelling material into the jet from the accretion inflow, parametrized in the quantity $\sigma_{M}$ as defined in [32].

Within each segment, the code operates in multiple-scattering mode (MIC)or in single-scattering mode (SIC) depending on the value of the Compton parameter, $y$, as described in [29]. We adopt the definition given by [30], which is

$$
y_{i}=A \max \left(\tau_{i}^{2}, \tau_{i}\right)
$$

where $\Theta_{i}=k_{\mathrm{B}} T_{e} /\left(m_{e} c^{2}\right)$ is the dimensionless electron temperature and $\tau_{i}$ is the Thompson optical depth of the $i$-th segment and $A=\left(16 \Theta_{i}^{2}+4 \Theta_{i}+1\right)$ is the amplification factor. Due to the assumptions made within AGNJET, the code is suitable for fitting low luminosity sources with emission from a relativistic jet, and we found ourselves in the domain where $A$ is large and $\tau_{i} \ll 1$. Therefore, if multiple scatterings are important, only the first few orders $(1<k<3-5)$ might be relevant, as shown in the left panel of Fig. 2. Considering also that we want to optimize the computational time as much as possible, we stop the iterations at $k=10$ (see e.g. [31]). However, we are still completing the tests and working on alternative possibilities for the convergence criterion.

As for the pairs, at present, we provide a comparison of the lepton number density, $n$, photon number density, $n_{\mathrm{ph}}$, pair annihilation rate, $\dot{n}_{\mathrm{pa}} \sim\langle\overline{v \sigma}\rangle n^{2}$, and pair production rate, $\dot{n}_{\mathrm{pp}} \sim \sigma_{\mathrm{T}} c n_{\mathrm{ph}}^{2}$, for the first few segments of the jet and use it as flag to interpret our best fit parameter values. However, efforts are being put into implementing a more self-consistent treatment of such warnings.

Most recently, we have been working on a new, and much more physically self-consistent dynamical model for jets. We are in the process of implementing a new algorithm to solve $r$-selfsimilar RMHD equations describing the morphology and the dynamics of relativistic jets. Starting from the equations given in [6], in CC17a we calculated the exact contributions of the gravitational potential (for a Newtonian and a Paczyńsky-Wiita potential) in the gravity terms, and we developed a new scheme for the integration of the system. We show that the correction of the gravitational 
potential functions significantly affects several properties of the solutions, particularly the geometry of the jet. From a partial exploration of the highly dimensional parameter space, we cannot yet infer general characteristics of jet solutions for any value and combination of the free parameters. However we can map and label the solutions we have so far to determine the strategy for current and future studies, targeted to applications to astrophysical objects (Fig. 2, right panel) in combination with AGNJET .

\section{Conclusions}

Jets are not only an extremely interesting and widespread phenomenon in the universe, but they also appear to play a fundamental role in the systems that are hosting them. Unveiling the physics behind relativistic outflows is a complex task and it requires accurate and comprehensive GRMHD and RT modelling. However complex GRMHD simulations are still a step behind when a direct test against observations is needed, due to both the absence of radiation processes and a prohibitive computational time at least in the nearby future. Coupling semi-analytical RMHD and RT is thus a key method for exploring physical parameter space and guiding the progress made in the field of simulations, while progresses are made in the simulation field (e.g [33]). Recently, we improved AGNJET by relaxing some of its basic assumptions, including additional radiative transfer processes and refining the ones that were already there. Currently, we are finding, studying and classifying RMHD jet solutions which will be used to substitute the geometry and the dynamics of AGNJET, according to the specific source we will be investigating.

\section{References}

[1] S. Markoff, M. A. Nowak, and J. Wilms, "Going with the Flow: Can the Base of Jets Subsume the Role of Compact Accretion Disk Coronae?," Astrophys. J., vol. 635, pp. 1203-1216, Dec. 2005.

[2] C. Ceccobello and et al. Mon. Not. R. Astron. Soc., 2017b, submitted.

[3] R. M. T. Connors, S. Markoff, M. A. Nowak, J. Neilsen, C. Ceccobello, P. Crumley, C. S. Froning, E. Gallo, and J. E. Nip, "Mass-scaling as a method to constrain outflows and particle acceleration from low-luminosity accreting black holes," ArXiv e-prints, Dec. 2016.

[4] P. Polko, D. L. Meier, and S. Markoff, "Determining the Optimal Locations for Shock Acceleration in Magnetohydrodynamical Jets,” Astrophys. J., vol. 723, pp. 1343-1350, Nov. 2010.

[5] P. Polko, D. L. Meier, and S. Markoff, "Linking accretion flow and particle acceleration in jets - I. New relativistic magnetohydrodynamical jet solutions including gravity," Mon. Not. R. Astron. Soc., vol. 428, pp. 587-598, Jan. 2013.

[6] P. Polko, D. L. Meier, and S. Markoff, "Linking accretion flow and particle acceleration in jets - II. Self-similar jet models with full relativistic MHD gravitational mass," Mon. Not. R. Astron. Soc., vol. 438, pp. 959-970, Feb. 2014.

[7] C. Ceccobello and et al. Mon. Not. R. Astron. Soc., 2017a, submitted.

[8] E. Gallo, R. Fender, C. Kaiser, D. Russell, R. Morganti, T. Oosterloo, and S. Heinz, “A dark jet dominates the power output of the stellar black hole Cygnus X-1," Nature, vol. 436, pp. 819-821, Aug. 2005. 
[9] A. C. Fabian, "Observational Evidence of Active Galactic Nuclei Feedback," Ann. Rev. Astron. Astrophys., vol. 50, pp. 455-489, Sept. 2012.

[10] R. P. Fender, "Powerful jets from black hole X-ray binaries in low/hard X-ray states," Mon. Not. R. Astron. Soc., vol. 322, pp. 31-42, Mar. 2001.

[11] E. G. Körding, S. Jester, and R. Fender, "Accretion states and radio loudness in active galactic nuclei: analogies with X-ray binaries,” Mon. Not. R. Astron. Soc., vol. 372, pp. 1366-1378, Nov. 2006.

[12] A. Merloni, S. Heinz, and T. di Matteo, "A Fundamental Plane of black hole activity," Mon. Not. R. Astron. Soc., vol. 345, pp. 1057-1076, Nov. 2003.

[13] H. Falcke, E. Körding, and S. Markoff, "A scheme to unify low-power accreting black holes. Jet-dominated accretion flows and the radio/X-ray correlation," Astron. Astrophys., vol. 414, pp. 895-903, Feb. 2004.

[14] R. M. Plotkin, S. Markoff, B. C. Kelly, E. Körding, and S. F. Anderson, "Using the Fundamental Plane of black hole activity to distinguish X-ray processes from weakly accreting black holes," Mon. Not. R. Astron. Soc., vol. 419, pp. 267-286, Jan. 2012.

[15] J. C. McKinney, "General relativistic magnetohydrodynamic simulations of the jet formation and large-scale propagation from black hole accretion systems," Mon. Not. R. Astron. Soc., vol. 368, pp. 1561-1582, June 2006.

[16] G. E. Romero, D. F. Torres, M. M. Kaufman Bernadó, and I. F. Mirabel, "Hadronic gamma-ray emission from windy microquasars," Astron. Astrophys., vol. 410, pp. L1-L4, Oct. 2003.

[17] F. Yuan, W. Cui, and R. Narayan, "An Accretion-Jet Model for Black Hole Binaries: Interpreting the Spectral and Timing Features of XTE J1118+480," Astrophys. J., vol. 620, pp. 905-914, Feb. 2005.

[18] A. A. Zdziarski, P. Lubiński, and M. Sikora, "The MeV spectral tail in Cyg X-1 and optically thin emission of jets," Mon. Not. R. Astron. Soc., vol. 423, pp. 663-675, June 2012.

[19] A. A. Zdziarski, Ł. Stawarz, P. Pjanka, and M. Sikora, "Jet models for black hole binaries in the hard spectral state," Mon. Not. R. Astron. Soc., vol. 440, pp. 2238-2254, May 2014.

[20] S. Markoff, H. Falcke, and R. Fender, "A jet model for the broadband spectrum of XTE J1118+480. Synchrotron emission from radio to X-rays in the Low/Hard spectral state," Astron. Astrophys., vol. 372, pp. L25-L28, June 2001.

[21] H. Falcke and P. L. Biermann, "The jet-disk symbiosis. I. Radio to X-ray emission models for quasars.," Astron. Astrophys., vol. 293, Jan. 1995.

[22] H. Falcke, “The Nuclear Jet in M81,” Astrophys. J., Lett., vol. 464, p. L67, June 1996.

[23] H. Falcke and S. Markoff, "The jet model for Sgr A*: Radio and X-ray spectrum," Astron. Astrophys., vol. 362, pp. 113-118, Oct. 2000.

[24] D. Maitra, S. Markoff, C. Brocksopp, M. Noble, M. Nowak, and J. Wilms, "Constraining jet/disc geometry and radiative processes in stellar black holes XTE J1118+480 and GX 339-4," Mon. Not. R. Astron. Soc., vol. 398, pp. 1638-1650, Oct. 2009.

[25] R. M. Plotkin, E. Gallo, S. Markoff, J. Homan, P. G. Jonker, J. C. A. Miller-Jones, D. M. Russell, and S. Drappeau, "Constraints on relativistic jets in quiescent black hole X-ray binaries from broad-band spectral modelling," Mon. Not. R. Astron. Soc., vol. 446, pp. 4098-4111, Feb. 2015.

[26] S. Markoff, M. A. Nowak, E. Gallo, R. Hynes, J. Wilms, R. M. Plotkin, D. Maitra, C. V. Silva, and S. Drappeau, "As Above, So Below: Exploiting Mass Scaling in Black Hole Accretion to Break Degeneracies in Spectral Interpretation," Astrophys. J., Lett., vol. 812, p. L25, Oct. 2015. 
[27] J. Malzac, "The spectral energy distribution of compact jets powered by internal shocks," Mon. Not. R. Astron. Soc., vol. 443, pp. 299-317, Sept. 2014.

[28] G. R. Blumenthal and R. J. Gould, "Bremsstrahlung, Synchrotron Radiation, and Compton Scattering of High-Energy Electrons Traversing Dilute Gases," Reviews of Modern Physics, vol. 42, pp. 237-271, 1970.

[29] G. B. Rybicki and A. P. Lightman, Radiative processes in astrophysics. 1979.

[30] G. Ghisellini, Synchrotron Self-Compton, pp. 89-93. Heidelberg: Springer International Publishing, 2013.

[31] L. A. Pozdnyakov, I. M. Sobol, and R. A. Syunyaev, "Comptonization and the shaping of X-ray source spectra - Monte Carlo calculations," Astrophysics and Space Physics Reviews, vol. 2, pp. 189-331, 1983.

[32] F. C. Michel, “Relativistic Stellar-Wind Torques,” Astrophys. J., vol. 158, p. 727, Nov. 1969.

[33] S. M. Ressler, A. Tchekhovskoy, E. Quataert, M. Chandra, and C. F. Gammie, "Electron thermodynamics in GRMHD simulations of low-luminosity black hole accretion," Mon. Not. R. Astron. Soc., vol. 454, pp. 1848-1870, Dec. 2015. 